\title{
Long-term clinical outcomes in type 1 Gaucher disease following 10 years of imiglucerase treatment
}

\author{
Neal J. Weinreb • Jack Goldblatt • Jacobo Villalobos • \\ Joel Charrow - J. Alexander Cole • Marcelo Kerstenetzky • \\ Stephan vom Dahl • Carla Hollak
}

Received: 14 March 2012 /Revised: 27 July 2012 / Accepted: 30 July 2012 /Published online: 14 September 2012

(C) The Author(s) 2012. This article is published with open access at Springerlink.com

\begin{abstract}
Objective We studied the effect of long-term alglucerase/ imiglucerase (Ceredase ${ }^{\circledR} /$ Cerezyme $^{\circledR}$, Genzyme, a Sanofi company, Cambridge, MA, USA) treatment on hematological, visceral, and bone manifestations of Gaucher disease type 1 (GD1).

Methods The International Collaborative Gaucher Group (ICGG) Gaucher Registry identified GD1 patients treated with alglucerase/imiglucerase who had dose and clinical data at first infusion and after 10 years of follow-up. Data for hemoglobin, platelet count, organ volumes, bone pain, and bone crisis were analyzed. Tests of the null hypothesis (no change from first infusion to 10 years) were performed using $t$ tests for within-patient absolute change in continuous measurements and $\mathrm{McNemar} / \mathrm{chi}$-square tests for
\end{abstract}

Communicated by: Gregory M. Pastores

Electronic supplementary material The online version of this article (doi:10.1007/s10545-012-9528-4) contains supplementary material, which is available to authorized users.

\section{N. J. Weinreb $(\bowtie)$}

University Research Foundation for Lysosomal Storage Diseases, Inc, Northwest Oncology Hematology Associates PA, 8170 Royal Palm Boulevard, Coral Springs, FL 33065, USA

e-mail: boneal@winning.com

\section{J. Goldblatt}

Genetic Services \& Familial Cancer Program of Western Australia, School of Pediatrics and Child Health, University of Western Australia, Perth, WA, Australia

J. Villalobos

Universidad Central de Venezuela, Caracas, Venezuela

\section{J. Charrow}

Division of Genetics, Birth Defects and Metabolism, Ann and Robert H. Lurie Children's Hospital of Chicago, Chicago, IL, USA change in distributions using categorical values. An alpha level of 0.05 designated statistical significance.

Results As of October 2011, 557 nonsplenectomized and 200 splenectomized patients met the inclusion criteria. The majority of GD1 patients had at least one N370S allele. Compared with nonsplenectomized patients at first infusion, splenectomized patients had lower percentages of anemia (26.0\% vs. $42.8 \%$ ) and thrombocytopenia (14.2\% vs. $76.3 \%$ ), similar percentages of moderate or severe hepatomegaly $(81.2 \%$ vs. $80.0 \%)$, and higher percentages of bone pain $(88.9 \%$ vs. $52.4 \%)$ and bone crises (38.3\% vs. $16.0 \%)$. After 10 years, both groups showed significant $(p<0.05)$ improvements in mean hemoglobin levels, platelet count, liver, and spleen (nonsplenectomized) volumes, and bone crises. Initial dosing in both groups ranged from $<15 \mathrm{U} / \mathrm{kg}$ to $\leq 90 \mathrm{U} / \mathrm{kg}$ every

J. A. Cole
Genzyme, a Sanofi company, Cambridge, MA, USA

M. Kerstenetzky

Instituto de Medicina Integral Professor Fernando Figueira, Recife, Pernambuco, Brazil

S. vom Dahl

Klinik für Innere Medizin, St. Franziskus-Hospital, Cologne,

Germany

C. Hollak

Department of Internal Medicine, Division of Endocrinology and Metabolism, Academic Medical Center, University of Amsterdam, Amsterdam, The Netherlands 
2 weeks. After 10 years, the majority was receiving 15 to $\leq 45$ $\mathrm{U} / \mathrm{kg}$ every 2 weeks.

Conclusion Ten years of imiglucerase treatment results in sustainable improvements in all GD1 parameters.

\section{Introduction}

Gaucher disease (GD) is an autosomal recessive lysosomal storage disorder that results from loss of function of acid $\beta$ glucosidase (EC 3.2.1.45; lysosomal glucocerebrosidase) due to mutations in the glucocerebrosidase gene, GBA1 (Grabowski et al. 2010). Deficient glucocerebrosidase activity leads to accumulation of the enzyme's substrate, glucocerebroside, in tissue macrophages and to displacement of normal cells in tissues of multiple organs, such as liver, spleen, and bone marrow (Charrow et al. 2000). Clinical manifestations of GD include hematological abnormalities (thrombocytopenia, anemia, leukopenia), splenic and hepatic enlargement (with associated dysfunction), skeletal disease (chronic bone pain, acute bone crises, defective bone mineralization, infarction, osteonecrosis, osteolysis, and pathological fractures) (Charrow et al. 2000; Weinreb et al. 2008), growth retardation (Kaplan et al. 2006), and decreased health-related quality of life (HR-QoL) (Weinreb et al. 2007). The traditional classification of GD recognizes three clinically distinct forms: type 1 (nonneuronopathic, OMIM \#230800), type 2 (acute neuronopathic, OMIM \#230900), and type 3 (chronic neuronopathic, OMIM \#231000). GD type 1 (GD1) is differentiated from GD2 and GD3 by the absence of overt, early-onset neurological signs and symptoms (Grabowski et al. 2010). However, distinct neurological symptoms such as peripheral neuropathy and Parkinson's disease may occur in GD1 (Biegstraaten et al. 2008).

GD1 was the first lysosomal storage disease to be successfully treated with enzyme therapy, initially in 1991 with placental-derived glucocerebrosidase, (alglucerase, Ceredase $^{\circledR}$, Genzyme Corporation, ${ }^{1}$ Cambridge, MA, USA), and beginning in 1994, with recombinant glucocerebrosidase, (imiglucerase, Cerezyme ${ }^{\circledR}$, Genzyme). Imiglucerase is highly effective in reversing visceral and hematologic manifestations of GD as well as some aspects of skeletal disease (Andersson et al. 2005; Barton et al. 1991; Charrow et al. 2007; Grabowski et al. 1998; Sims et al. 2008; Weinreb et al. 2008; Weinreb et al. 2002; Wenstrup et al. 2007) over a wide range of doses (Grabowski et al. 2009).

Evaluation of hematological and organ responses and skeletal manifestations is the standard minimum criterion for evaluating efficacy of treatment for GD1 in both clinical

${ }^{1}$ In 2011, Genzyme Corporation became Genzyme, a Sanofi company. trials and in everyday practice. This report used observational data from the International Collaborative Gaucher Group (ICGG) Gaucher Registry to analyze responses of GD1 patients at first infusion and after 10 years of imiglucerase treatment. We hypothesized that imiglucerase therapy would demonstrate a sustained benefit, even after 10 years of treatment.

\section{Materials and methods}

International Collaborative Gaucher Group (ICGG) Gaucher Registry

The ICGG Gaucher Registry was launched in 1991 to track clinical, demographic, genetic, biochemical, and therapeutic characteristics of patients with GD throughout the world, irrespective of disease severity, treatment status, or treatment choice (Charrow et al. 2000). Governance and scientific direction is provided by an international group of physician experts in GD, with operational support from Genzyme, a Sanofi company (Cambridge, MA, USA). With Institutional Review Board (IRB)/Ethics Committee approvals and after obtaining informed patient consent, physicians from 62 countries voluntarily submitted deidentified data on more than 6,000 patients, capturing 54,000 patient years of follow-up.

\section{Study population}

We identified all GD1 patients in the ICGG Gaucher Registry as of October 2011 who received imiglucerase (Cerezyme $^{\circledR}$, Genzyme) or alglucerase (Ceredase ${ }^{\circledR}$, Genzyme), which are therapeutically equivalent treatments (Grabowski et al. 1995). In the report presented here, the term imiglucerase is used to denote patients who received either alglucerase or imiglucerase. Inclusion criteria for the study data set were patients with GD1 who had dose and clinical data at first imiglucerase infusion (baseline) and after 10 years of therapy. Within the study cohort, subsets of patients were identified with clinical data for hemoglobin, platelet count, liver volume, spleen volume, bone pain, and bone crisis at treatment initiation (baseline) and after 10 years of followup. Patients without clinical data at baseline and after 10 years were excluded.

Because splenectomy is known to have an immediate and persistent influence on GD pathophysiology and patient phenotype, osteonecrosis (Deegan et al. 2011; Mistry et al. 2009), and hematological response (Cox et al. 2008; Hughes et al. 2007), data were further analyzed by splenectomy status. Patients were stratified into nonsplenectomized and splenectomized groups. Because the postprocedure phenotype after partial splenectomy is generally closer to that of 
patients with fully intact spleens than to those with total splenectomy, in this treatment response analysis, the small number of eligible partial splenectomy patients $(N=27)$ were included among the much larger number of patients with fully intact spleens $(N=557)$.

For this analysis, baseline was defined as a window of time ranging from 12 months prior to 1 month following the date of treatment initiation. The 10-year interval was defined as the time from 9.5 years to 10.5 years. Within each time window, whenever a patient had more than one clinical assessment date, the value associated with the date closest to the date of treatment initiation or the date closest to the 10 -year interval was incorporated into the analysis. No data were included after the Cerezyme manufacturing interruption (25 June 2009). If patients switched to an alternative form of enzyme replacement therapy, their data were excluded from the analysis.

Statistical analysis

Demographics and clinical parameters at treatment initiation and after 10 years (hemoglobin, platelet count, liver volume, spleen volume, bone pain, and bone crisis), and frequency of genotypes were characterized using descriptive statistics. Organ volumes, measured by magnetic resonance imaging (MRI), computed tomography (CT), or ultrasound (US), are reported in multiples of normal (MN) size predicted for body weight: approximately $2.5 \%$ of body weight for liver, and $0.2 \%$ for spleen (Elstein et al. 1997; Ludwig 1979). Clinical parameters were analyzed both as continuous measurements, and as categorical measurements using the following cut points:

- Anemia is defined according to age and gender norms for hemoglobin concentrations, as follows: $<12 \mathrm{~g} / \mathrm{dl}$ for males $>12$ years; $<11 \mathrm{~g} / \mathrm{dl}$ for females $>12$ years; $<10.5 \mathrm{~g} / \mathrm{dl}$ for children ages $>2-12$ years; $<9.5 \mathrm{~g} / \mathrm{dl}$ for children ages 6 months to 2 years; $<10.1 \mathrm{~g} / \mathrm{dl}$ for children younger than 6 months of age.

- Thrombocytopenia is categorized as mild or none $\left(\geq 120 \times 10^{3} / \mathrm{mm}^{3}\right)$, moderate $\left(60\right.$ to $\left.<120 \times 10^{3} / \mathrm{mm}^{3}\right)$, or severe $\left(<60 \times 10^{3} / \mathrm{mm}^{3}\right)$.

- Splenomegaly (spleen volume in multiples of normal) is scored as mild or none $(\leq 5)$, moderate $(>5$ to $\leq 15)$, or severe $(>15)$.

- Hepatomegaly (liver volume in multiples of normal) is scored as mild or none $(\leq 1.25)$, moderate $(>1.25$ to $\leq 2.5)$, or severe $(>2.5)$.

- Bone pain is defined as being present if the patient reported this event as occurring in the 30-day interval before the medical visit.

- Bone crisis was defined as present if the patient reported this event at the time of the medical visit. The ICGG Gaucher Registry defines bone crisis as: "Pain with acute onset that requires immobilization of the affected area, narcotics for the relief of pain, and may be accompanied by 1 or more of the following: periosteal elevation: elevated white blood cell count, fever, or debilitation $>3$ days."

- Doses used were those reported at first infusion and at 10 years.

For analysis of continuous values, we calculated both the within-patient absolute change and percentage change from treatment initiation to 10 years. For categorical analysis, we produced shift tables representing change in the distribution of values from first infusion to 10 years. The normality of the distributions was tested, and paired $t$ tests were performed. Tests of the null hypotheses (i.e. no change from first infusion to 10 years) were calculated using the paired $t$ test for the within-patient absolute change in continuous measurements, the McNemar test for binary variables, and the chi-square test when a variable had more than two categories. An alpha level of 0.05 denotes statistical significance.

\section{Results}

As of October 2011, 757 GD1 patients enrolled in the ICGG Gaucher Registry met the study inclusion criteria of receiving imiglucerase and having both clinical data and dose data at first infusion (baseline) and after 10 years of follow-up. Of these, 226 patients received imiglucerase only, and 531 started treatment with alglucerase and transitioned to imiglucerase. Of the overall patient cohort, 200 were splenectomized and 557 were not.

\section{Nonsplenectomized patients}

The demographic characteristics of nonsplenectomized GD1 patients are shown in Table 1. Most patients were diagnosed early in life (median age at diagnosis: 11 years). The median age for first imiglucerase infusion was not until 24 years. Genotypes were reported for approximately $89 \%$ of nonsplenectomized patients (Table 2). Nearly all (92\%) had at least one N370S allele (27\% homozygous, $65 \%$ heteroallelic). Among patients with available baseline data, prior to starting imiglucerase treatment, $42.8 \%$ of nonsplenectomized patients were anemic, $76.3 \%$ had moderate or severe thrombocytopenia, $86.9 \%$ had moderate or severe splenomegaly, $80.0 \%$ had moderate or severe hepatomegaly, $52.4 \%$ reported bone pain, and $16.0 \%$ reported bone crises (Table 4; Supplementary Figs. 1-6).

Changes in hematological, visceral organ, and skeletal characteristics reported at first infusion and after 10 years of treatment are shown in Table 3. For mean hemoglobin concentration $(\mathrm{g} / \mathrm{dl})$, there was a significant improvement 
Table 1 Demographic characteristics of nonsplenectomized and splenectomized patients with type 1 Gaucher disease

\begin{tabular}{|c|c|c|}
\hline & Nonsplenectomized $^{\mathrm{a}}$ & Splenectomized \\
\hline Patients Enrolled & $N=557$ & $N=200$ \\
\hline Sex, $N(\%)$ & $N=557$ & $N=200$ \\
\hline Males & $263(47)$ & $80(40)$ \\
\hline Females & $294(53)$ & $120(60)$ \\
\hline Age at Diagnosis ${ }^{\mathbf{b}}$ (years) & $N=554$ & $N=196$ \\
\hline Median (25th, 75th) & $11(5,30)$ & $10(4,23)$ \\
\hline Mean (SD) & $18(16)$ & $15(13)$ \\
\hline Min, Max & $<0^{\mathrm{c}}, 75$ & 0,53 \\
\hline Age at Diagnosis ${ }^{\mathrm{b}}, N(\%)$ & $N=554$ & $N=196$ \\
\hline Prenatal $^{\mathrm{c}}$ to $<10$ Years & $261(47)$ & $95(48)$ \\
\hline 10 to $<20$ years & $76(14)$ & $39(20)$ \\
\hline 20 to $<30$ years & $81(15)$ & $33(17)$ \\
\hline 30 to $<40$ years & $60(11)$ & $18(9)$ \\
\hline 40 to $<50$ years & $50(9)$ & $9(5)$ \\
\hline 50 to $<60$ years & $20(4)$ & $2(1)$ \\
\hline 60 to $<70$ years & $5(1)$ & 0 \\
\hline$\geq 70$ years & $1(<1)$ & 0 \\
\hline Age at First infusion (years) & $N=557$ & $N=200$ \\
\hline Median (25th, 75th) & $24(9,42)$ & $38(26,49)$ \\
\hline Mean (SD) & $26(19)$ & $37(16)$ \\
\hline Min, Max & 0,75 & 3,74 \\
\hline Age at Last Follow-up (years) & $N=557$ & $N=200$ \\
\hline Median (25th, 75th) & $37(23,56)$ & $54(40,63)$ \\
\hline Mean (SD) & 40 (19) & $52(16)$ \\
\hline Min, Max & 13,87 & 18,87 \\
\hline
\end{tabular}

$S D$ standard deviation

${ }^{a}$ Includes 27 patients with partial splenectomy

${ }^{\mathrm{b}}$ Patients with no diagnosis date or with diagnosis date earlier than 1 year prior to birth were excluded from the analysis

${ }^{\mathrm{c}}$ Diagnosed prenatally

observed at 10 years (11.2 increased to $13.6 ; P<0.0001)$ compared with the start of treatment. Approximately $88 \%$ of nonsplenectomized patients who were anemic at first infusion were no longer so after 10 years of treatment (Table 4, Supplementary Fig. 1a).

Nonsplenectomized patients had a mean platelet count of $95.3 \times 10^{3} / \mathrm{mm}^{3}$ at first infusion, which improved to a mean of $166.6 \times 10^{3} / \mathrm{mm}^{3}$ after 10 years $(P<0.0001)$ (Table 3$)$. At 10 years, the number of patients with severe thrombocytopenia decreased significantly $(P<0.001)$ (Supplementary Fig. 2a). The 397 patients eligible for platelet response analysis included 20 with partial splenectomies $(5.0 \%)$. Mean platelet count in these patients improved from $148 \times 10^{3} / \mathrm{mm}^{3}$ at first infusion to $190 \times 10^{3} / \mathrm{mm}^{3}$ after 10 years (data not shown). Nonsplenectomized GD1 patients $(N=107)$ demonstrated significant $(P<0.0001)$ improvement in spleen size
Table 2 Frequency of genotype categories of nonsplenectomized and splenectomized patients with type 1 Gaucher disease

\begin{tabular}{|c|c|c|}
\hline Genotype, $N(\%)$ & $\begin{array}{l}\text { Nonsplenectomized }^{\mathrm{c}} \\
(N=493)\end{array}$ & $\begin{array}{l}\text { Splenectomized } \\
(N=189)\end{array}$ \\
\hline N370S/N370S & $132(27)$ & $25(13)$ \\
\hline N370S/L444P & $106(22)$ & $37(20)$ \\
\hline N370S/rare allele ${ }^{a}$ & $93(19)$ & $26(14)$ \\
\hline $\mathrm{N} 370 \mathrm{~S} / ?^{\mathrm{b}}$ & $52(11)$ & $27(14)$ \\
\hline N370S/84GG & $51(10)$ & $30(16)$ \\
\hline N370S/IVS2+1 & $17(3)$ & $10(5)$ \\
\hline L444P/rare allele ${ }^{\mathrm{a}}$ & $11(2)$ & $8(4)$ \\
\hline Rare allele $/$ rare allele ${ }^{\mathrm{a}}$ & $11(2)$ & $10(5)$ \\
\hline L444P/L444P & $8(2)$ & $4(2)$ \\
\hline $\mathrm{L} 444 \mathrm{P} / ?^{\mathrm{b}}$ & $3(1)$ & $7(4)$ \\
\hline D409H/rare allele ${ }^{\mathrm{a}}$ & $2(<1)$ & - \\
\hline$?^{\mathrm{b}} / ?^{\mathrm{b}}$ & $2(<1)$ & $1(1)$ \\
\hline L444P/D409H & $1(<1)$ & - \\
\hline D409H/D409H & $1(<1)$ & - \\
\hline 84GG/rare allele ${ }^{\mathrm{a}}$ & $1(<1)$ & $2(1)$ \\
\hline IVS $2+1 /$ rare allele $^{\mathrm{a}}$ & $1(<1)$ & $1(1)$ \\
\hline Rare allele ${ }^{\mathrm{a}} / ?^{\mathrm{b}}$ & $1(<1)$ & - \\
\hline $84 \mathrm{GG} / ?^{\mathrm{b}}$ & - & $1(1)$ \\
\hline
\end{tabular}

${ }^{\text {a }}$ Rare allele is defined as known allele that is not N370S, L444P, IVS2 +1 , D409H, or $84 \mathrm{GG}$

${ }^{\mathrm{b}}$ Results of genotype test did not match any tested mutations

${ }^{\mathrm{c}}$ Includes 27 patients with partial splenectomy

over the 10 years of treatment. Mean spleen volume at first infusion was 19.4 MN and decreased to a mean of 5.2 MN after 10 years (Table 3 ). The number of patients with moderate and severe splenomegaly was significantly lower $(P<0.0001)$ after 10 years of imiglucerase therapy (Table 4, Supplementary Fig. 3). Only three partial splenectomy patients had sufficient data for inclusion in this analysis (mean spleen volume at first infusion: $9.0 \mathrm{MN}$; after 10 years: $3.5 \mathrm{MN}$; data not shown). There were also significant $(P<0.0001)$ reductions in liver size over 10 years of treatment (Table 3 ). At first infusion, the mean liver volume for nonsplenectomized patients was $1.8 \mathrm{MN}$ and decreased to $1.0 \mathrm{MN}$ after 10 years. The numbers of patients with moderate and severe hepatomegaly decreased significantly $(P<0.0001)$ after 10 years; no patients had severe hepatomegaly at this time point (Table 4, Supplementary Fig. 4a).

Imiglucerase treatment positively affected skeletal symptoms, as noted by decreased bone pain (Table 4, Supplementary Fig. 5a) and bone crises (Table 4, Supplementary Fig. 6a). For nonsplenectomized GD1 patients with bone pain data, 98/ $187(52.4 \%)$ reported bone pain prior to initiation of imiglucerase (Table 4, Supplementary Fig. 5a). Of these, $57.1 \%(n=$ 56) no longer reported bone pain after 10 years of treatment. Of the 89 patients with no reported bone pain prior to initiation 
Table 3 Change in clinical parameters from initiation of imiglucerase to 10 years following first infusion in nonsplenectomized and splenectomized patients with type 1 Gaucher disease

\begin{tabular}{|c|c|c|c|c|c|c|}
\hline \multicolumn{4}{|c|}{ Nonsplenectomized $^{\mathrm{a}}$} & \multicolumn{3}{|l|}{ Splenectomized } \\
\hline Parameter & First Infusion & $\begin{array}{l}10 \text { Years Following } \\
\text { First Infusion }\end{array}$ & $\begin{array}{l}\text { Calculated } \\
\text { Change }\end{array}$ & First Infusion & $\begin{array}{l}10 \text { Years Following } \\
\text { First Infusion }\end{array}$ & $\begin{array}{l}\text { Calculated } \\
\text { Change }\end{array}$ \\
\hline \multicolumn{7}{|c|}{ Hemoglobin $(\mathrm{g} / \mathrm{dl})$} \\
\hline No. & 376 & 376 & 376 & 131 & 131 & 131 \\
\hline Mean (SD) & $11.2(1.7)$ & $13.6(1.6)$ & $2.4(1.8)$ & $11.9(1.7)$ & $13.4(1.6)$ & $1.5(1.8)$ \\
\hline Median & 11.2 & 13.6 & 2.4 & 12.0 & 13.5 & 1.4 \\
\hline Min, Max & $6.7,16.2$ & $8.8,17.9$ & $-3.0,7.9$ & $6.8,16.9$ & $6.9,17.6$ & $-5.0,7.4$ \\
\hline$P$ value* & & & $<0.0001$ & & & $<0.0001$ \\
\hline \multicolumn{7}{|c|}{ Platelet Count (x 10 $\left./ \mathrm{mm}^{3}\right)$} \\
\hline No. & 397 & 397 & 397 & 141 & 141 & 141 \\
\hline Mean (SD) & $95.3(52.8)$ & $166.6(59.7)$ & $71.3(51.9)$ & $237.8(125.5)$ & $311.2(98.3)$ & $73.4(126.0)$ \\
\hline Median & 87.0 & 169.0 & 71.0 & 218.0 & 307.0 & 68.0 \\
\hline Min, Max & $16.0,448.0$ & $25.0,433.0$ & $-170.0,219.0$ & $6.0,726.0$ & $98.0,644.0$ & $-329.0,464.0$ \\
\hline$P$ value* & & & $<0.0001$ & & & $<0.0001$ \\
\hline \multicolumn{7}{|c|}{ Spleen Volume (Multiples of Normal) } \\
\hline No. & 107 & 107 & 107 & - & - & - \\
\hline Mean (SD) & $19.4(16.2)$ & $5.2(3.6)$ & $-14.3(15.0)$ & - & - & - \\
\hline Median & 15.9 & 3.7 & -10.7 & - & - & - \\
\hline Min, Max & $1.4,91.8$ & $1.2,15.7$ & $-88.8,1.6$ & - & - & - \\
\hline$P$ value* & & & $<0.0001$ & - & - & - \\
\hline \multicolumn{7}{|c|}{ Liver Volume (Multiples of Normal) } \\
\hline No. & 105 & 105 & 105 & 32 & 32 & 32 \\
\hline Mean (SD) & $1.8(0.8)$ & $1.0(0.2)$ & $-0.8(0.7)$ & $2.2(1.0)$ & $1.0(0.24)$ & $1.2(0.91)$ \\
\hline Median & 1.7 & 1.0 & -0.7 & 2.1 & 0.93 & -0.97 \\
\hline Min, Max & $0.6,6.0$ & $0.6,1.9$ & $-4.5,0.2$ & $0.7,4.4$ & $0.7,1.7$ & $-3.4,0.1$ \\
\hline$P$ value* & & & $<0.0001$ & & & $<0.0001$ \\
\hline
\end{tabular}

$S D$ standard deviation

${ }^{a}$ Includes 27 patients with partial splenectomy

*Tests of the null hypotheses (i.e. no change from first infusion to 10 years) were calculated using the paired t-test for the within-patient absolute change

of imiglucerase, 75 (84.3\%) continued to be pain free when evaluated after 10 years of therapy. For nonsplenectomized patients with bone crisis data, 27/169 (16.0\%) had a report of bone crisis prior to initiation of imiglucerase. Of these, $92.6 \%$ $(n=25)$ did not report a bone crisis after 10 years of treatment (Table 4, Supplementary Fig. 6a). None of the 142 patients who reported no bone crises prior to initiation of imiglucerase reported bone crises after 10 years of therapy. At initiation of treatment (Table 6), most patients were dosed in the middle range at either $>15$ to $\leq 45 \mathrm{U} / \mathrm{kg}$ every 2 weeks $(n=244$ patients, $43.8 \%$ ) or $>45$ to $\leq 90 \mathrm{U} / \mathrm{kg}$ every 2 weeks ( $n=$ $198,35.5 \%)$. A smaller percentage of patients received low doses ( $\leq 15 \mathrm{U} / \mathrm{kg}$ every 2 weeks, $n=112,20.1 \%)$ and hardly any received high doses ( $>90$ to $\leq 150 \mathrm{U} / \mathrm{kg}$ every 2 weeks, $n=3,<1 \%$ ). After 10 years of imiglucerase, most (82.2\%) were receiving imiglucerase doses in the middle range ( $>15$ to $\leq 45$ or $>45$ to $\leq 90 \mathrm{U} / \mathrm{kg}$ every 2 weeks), but now the majority
(57.6\%) were receiving $>15$ to $\leq 45 \mathrm{U} / \mathrm{kg}$ every 2 weeks, reflecting shifts away from lower and higher dose groups.

Splenectomized patients

Demographic characteristics of splenectomized patients are shown in Table 1. As with patients with intact spleens, most splenectomized patients were diagnosed early in life (median age at diagnosis 10 years). The splenectomized group differed from the nonsplenectomized group in having proportionally more females ( $60 \%$ vs. $53 \%$, respectively), a higher proportion of patients diagnosed from 10 to $<30$ years ( $37 \%$ vs. $29 \%$ ), and older median ages at first imiglucerase infusion (38 years vs. 24 years) and at last follow-up (54 years vs. 37 years). Genotypes were reported for approximately $95 \%$ of splenectomized patients (Table 2 ). The majority $(82 \%)$ had at least one N370S allele $(13 \%$ 
Table 4 Change in clinical parameters from initiation of imiglucerase to 10 years following first infusion in nonsplenectomized ${ }^{\mathrm{a}}$ patients with type 1 Gaucher disease

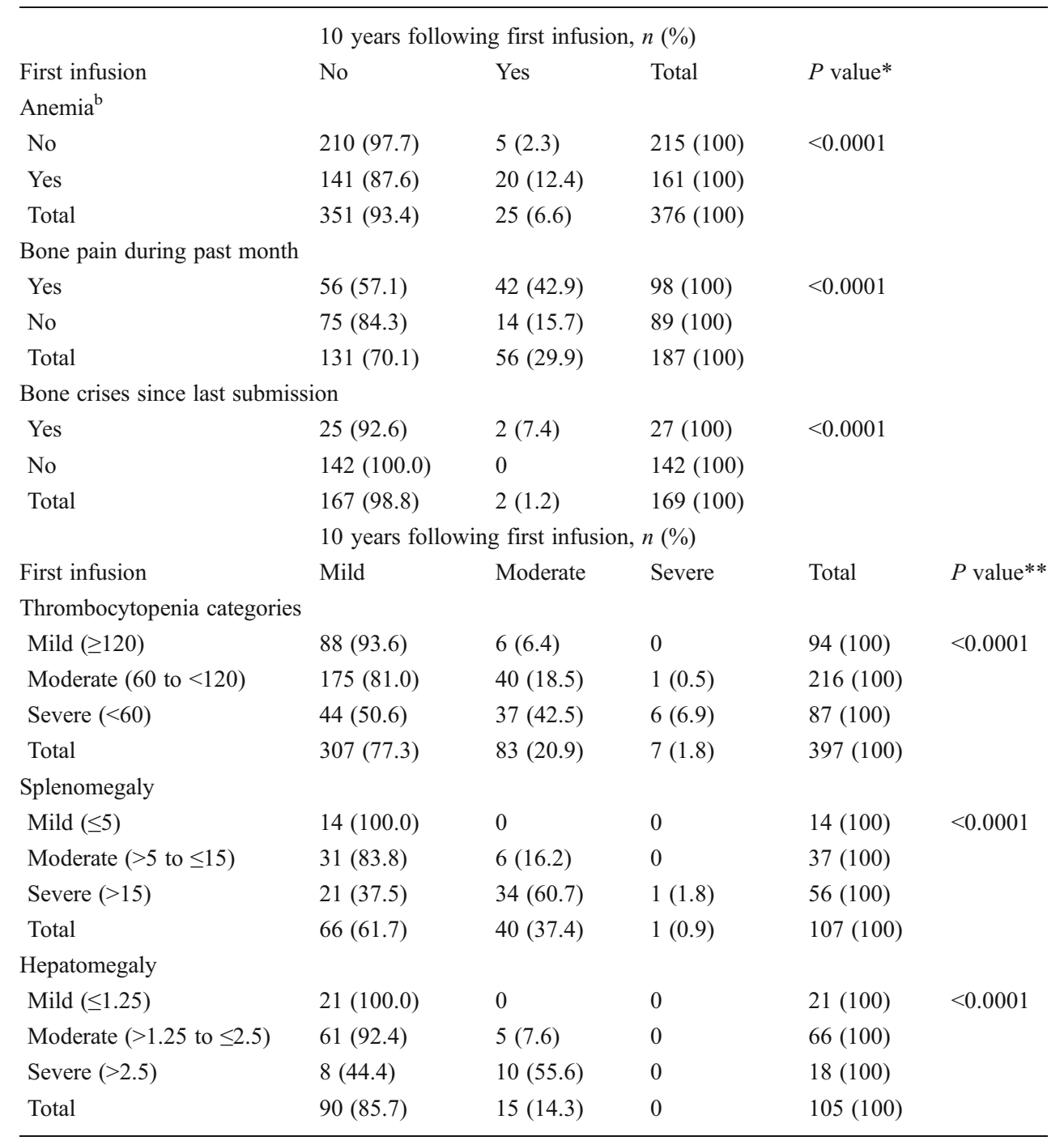

10 years following first infusion, $n(\%)$
${ }^{\mathrm{a}}$ Includes patients with partial splenectomy

${ }^{\mathrm{b}}$ Anemia is defined according to age and gender norms for hemoglobin concentrations, as follows: $<12 \mathrm{~g} / \mathrm{dl}$ for males older than 12 years; $<11 \mathrm{~g} / \mathrm{dl}$ for females older than 12 years;

$<10.5 \mathrm{~g} / \mathrm{dl}$ for children ages $>$

$2-12$ years; $<9.5 \mathrm{~g} / \mathrm{dl}$ for children ages 6 months to 2 years; $<10.1$

$\mathrm{g} / \mathrm{dl}$ for children younger than 6 months

*Tests of the null hypotheses (i.e., no change from first infusion to 10 years) were calculated using the McNemar test

**Tests of the null hypotheses (i.e., no change from first infusion to 10 years) were calculated using the chi- square test homozygous, $69 \%$ heteroallelic) and were less likely to have N370S homozygosity and more likely to lack an N370S allele than patients with intact spleens. Prior to starting imiglucerase treatment, splenectomized patients had the following clinical status at baseline: $26.0 \%$ were anemic, $14.2 \%$ had moderate or severe thrombocytopenia, $81.2 \%$ had moderate or severe hepatomegaly, $88.9 \%$ reported bone pain, and $38.3 \%$ reported bone crises (Table 5, Supplementary Figs. 1b, 2b, 4b, 5b, 6b). Compared with the nonsplenectomized group, there was a lower percentage of patients with anemia (26.0\% vs. $42.8 \%$ ) and thrombocytopenia (14.2\% vs. $76.3 \%$ ), a similar percentage with moderate or severe hepatomegaly $(81.2 \%$ vs. $80.0 \%)$, and a higher percentage with bone pain ( $88.9 \%$ vs. $52.4 \%$ ) and bone crises (38.3\% vs. $16.0 \%)$.

Changes in hematological, visceral organ, and skeletal characteristics for splenectomized patients reported at first infusion and after 10 years of treatment are shown in Table 3 . The patients demonstrated a significant $(P<0.0001)$ improvement in mean hemoglobin concentration $(\mathrm{g} / \mathrm{dl}) 10$ years following first infusion (from 11.9 to 13.4 ). In fact, $91.2 \%$ of patients who were anemic at first infusion were no longer so after 10 years of treatment (Table 5, Supplementary Fig. 1b). Platelet counts improved with treatment (mean at baseline $237.8 \times 10^{3} / \mathrm{mm}^{3}$ vs. mean at 10 years $311.2 \times 10^{3} / \mathrm{mm}^{3}$ ), as it did nonsplenectomized patients (Tables 3 and 5, Supplementary Fig. 2b). The number of nonsplenectomized patients with severe thrombocytopenia decreased significantly $(P<0.0001)$ after 10 years; no splenectomized patients had severe thrombocytopenia at 10 years (Table 5, Supplementary Fig. 2b). Liver volumes significantly improved $(P<0.001)$ in splenectomized patients after 10 years (Table 3 ), with mean volume decreasing from 2.2 $\mathrm{MN}$ to a median of 1.0 MN (Table 3). The response was similar to that of nonsplenectomized patients, who had less hepatomegaly prior to treatment (mean at first infusion $1.8 \mathrm{MN}$ decreased to a mean of $1.0 \mathrm{MN}$ at 10 years). The percentage of splenectomized patients reporting bone pain at first infusion was $88.9 \%$ (48/54), which decreased to $61.1 \%(33 / 54)$ after 
Table 5 Change in clinical parameters from initiation of imiglucerase to 10 years following first infusion in splenectomized patients with type 1 Gaucher disease

\section{$S D$ standard deviation}

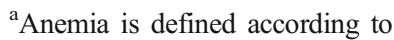
age and gender norms for hemoglobin concentrations, as follows: $<12 \mathrm{~g} / \mathrm{dl}$ for males $>12$ years; < $11 \mathrm{~g} / \mathrm{dl}$ for females $>12$ years; $<$ $10.5 \mathrm{~g} / \mathrm{dl}$ for children ages $>2-12$ years; $<9.5 \mathrm{~g} / \mathrm{dl}$ for children ages 6 months to 2 years; $<10.1 \mathrm{~g} / \mathrm{dl}$ for children $<6$ months

*Tests of null hypotheses (i.e., no change from first infusion to 10 years) were calculated using the McNemar test

**Tests of the null hypotheses (i.e., no change from first infusion to 10 years) were calculated using the chi-square test

\begin{tabular}{|c|c|c|c|c|c|}
\hline \multirow[b]{2}{*}{ First infusion } & \multicolumn{5}{|c|}{10 years following first infusion, $N(\%)$} \\
\hline & No & Yes & Total & $P$ value* & $P$ value** \\
\hline \multicolumn{6}{|l|}{ Anemia $^{\mathrm{a}}$} \\
\hline No & $94(96.9)$ & $3(3.1)$ & $97(100)$ & \multirow[t]{3}{*}{$<0.0001$} & \\
\hline Yes & $31(91.2)$ & $3(8.8)$ & $34(100)$ & & \\
\hline Total & $125(95.4)$ & $6(4.6)$ & 131(100) & & \\
\hline \multicolumn{6}{|l|}{ Bone Pain during past month } \\
\hline Yes & $18(37.5)$ & $30(62.5)$ & $48(100)$ & \multirow[t]{3}{*}{0.0011} & \\
\hline No & $3(50.0)$ & $3(50.0)$ & $6(100)$ & & \\
\hline Total & $21(38.9)$ & $33(61.1)$ & $54(100)$ & & \\
\hline \multicolumn{6}{|c|}{ Bone Crises since last submission } \\
\hline Yes & $15(83.3)$ & $3(16.7)$ & $18(100)$ & \multirow[t]{3}{*}{0.0001} & \\
\hline No & $29(100.0)$ & 0 & $29(100)$ & & \\
\hline \multirow[t]{2}{*}{ Total } & $44(93.6)$ & $3(6.4)$ & $47(100)$ & & \\
\hline & \multicolumn{5}{|c|}{10 years following first infusion, $N(\%)$} \\
\hline First infusion & Mild & Moderate & Severe & Total & \\
\hline \multicolumn{6}{|c|}{ Thrombocytopenia (platelet count $\times 10^{3} / \mathrm{mm}^{3}$ ) } \\
\hline Mild $(\geq 120)$ & $120(99.2)$ & $1(0.8)$ & 0 & $121(100)$ & \multirow[t]{4}{*}{0.9201} \\
\hline Moderate $(60$ to $<120)$ & $13(100.0)$ & 0 & 0 & $13(100)$ & \\
\hline Severe $(<60)$ & $7(100.0)$ & 0 & 0 & $7(100)$ & \\
\hline Total & $140(99.3)$ & $1(0.7)$ & 0 & $141(100)$ & \\
\hline \multicolumn{6}{|l|}{ Hepatomegaly } \\
\hline Mild $(\leq 1.25)$ & $6(100.0)$ & 0 & 0 & $6(100)$ & \multirow[t]{4}{*}{0.0273} \\
\hline Moderate $(>1.25$ to $\leq 2.5)$ & $16(88.9)$ & $2(11.1)$ & 0 & $18(100)$ & \\
\hline Severe $(>2.5)$ & $4(50.0)$ & $4(50.0)$ & 0 & $8(100)$ & \\
\hline Total & $26(81.3)$ & $6(18.8)$ & 0 & $32(100)$ & \\
\hline
\end{tabular}

10 years (Table 5, Supplementary Fig. 5b). The majority of patients without bone pain after 10 years were from the initial subgroup reporting bone pain (18/21, $85.7 \%$ ). Absence of bone pain prior to first infusion was reported for only six splenectomized patients, three of whom reported bone pain when evaluated after 10 years. Compared with nonsplenectomized patients, higher percentages reported bone pain prior to and after 10 years, irrespective of bone pain status prior to treatment. Treatment positively affected the frequency of bone crises in splenectomized patients (Table 5, Supplementary Fig. 6b), decreasing from $38.3 \%$ $(18 / 47)$ to $6.4 \%(3 / 47)$, all of whom were from the subgroup reporting bone crises prior to treatment. Similar to nonsplenectomized patients, at the start of treatment, the majority of splenectomized patients received imiglucerase in the middle two dose ranges: $>15$ to $\leq 45 \mathrm{U} / \mathrm{kg}$ every 2 weeks $(N=74,37.0 \%) ;>45$ to $\leq 90 \mathrm{U} / \mathrm{kg}$ every 2 weeks $(N=75,37.5 \%)$ (Table 6$)$. The remainder $(N=51$, $25.5 \%)$ received low doses ( $\leq 15 \mathrm{U} / \mathrm{kg}$ every 2 weeks), and no patient received high doses $(>90$ to $\leq 150 \mathrm{U} / \mathrm{kg}$ every 2 weeks). After 10 years, the majority of patients
$(N=107,53.5 \%)$ were receiving imiglucerase doses in the range of 15 to $\leq 45 \mathrm{U} / \mathrm{kg}$ every 2 weeks, reflecting a shift from other dose groups. The dose distribution at 10 years appeared similar to that of nonsplenectomized patients.

\section{Discussion}

The focus of this study, which analyzed the responses of GD1 patients at first infusion and after 10 years of imiglucerase treatment, was to assess whether responses to therapy were maintained for this time period. Clinical responses, including the influence of spleen status, were analyzed in a large cohort of nonsplenectomized $(n=557)$ and splenectomized $(n=200)$ GD1 patients enrolled in the ICGG Gaucher Registry. The results indicated that all parameters studied responded favorably to imiglucerase after 10 years of treatment.

The ICGG Gaucher Registry is a voluntary enterprise, and all registry data are analyzed retrospectively and unaudited by the study authors (Hollak et al. 2011). Data on each 
Table 6 Distribution of dose received ${ }^{\mathrm{a}}$ at first infusion and at 10 years following first infusion for patients with type 1 Gaucher disease, $N$ (\%)

Dosage distribution

\begin{tabular}{|c|c|c|c|c|c|c|}
\hline \multicolumn{2}{|l|}{ Nonsplenectomized $^{\mathrm{b}}$} & \multicolumn{5}{|c|}{10 years following first infusion } \\
\hline First infusion dose received (U/kg every 2 weeks) & $\leq 15$ & $>15 \leq 45$ & $>45 \leq 90$ & $>90 \leq 150$ & Total & $P$ value \\
\hline$\leq 15$ & $54(48.2)$ & $46(41.1)$ & $11(9.8)$ & $1(0.9)$ & $112(100)$ & $<0.0001$ \\
\hline$>15 \leq 45$ & $29(11.9)$ & $154(63.1)$ & $61(25.0)$ & 0 & $244(100)$ & \\
\hline$>45 \leq 90$ & $12(6.1)$ & $120(60.6)$ & $63(31.8)$ & $3(1.5)$ & $198(100)$ & \\
\hline$>90 \leq 150$ & 0 & $1(33.3)$ & $2(66.7)$ & 0 & $3(100)$ & \\
\hline Total & $95(17.1)$ & $321(57.6)$ & $137(24.6)$ & $4(0.7)$ & $557(100)$ & \\
\hline \multicolumn{2}{|l|}{ Splenectomized } & \multicolumn{5}{|c|}{10 years following first infusion } \\
\hline First infusion dose received (U/kg every 2 weeks) & $\leq 15$ & $>15 \leq 45$ & $>45 \leq 90$ & $>90 \leq 150$ & Total & $P$ value \\
\hline$\leq 15$ & $22(43.1)$ & $26(51.0)$ & $2(3.9)$ & $1(2.0)$ & $51(100)$ & $<0.0001$ \\
\hline$>15 \leq 45$ & $9(12.2)$ & $45(60.8)$ & $20(27.0)$ & 0 & 74 (100) & \\
\hline$>45 \leq 90$ & $5(6.7)$ & $36(48.0)$ & $32(42.7)$ & $2(2.7)$ & $75(100)$ & \\
\hline$>90 \leq 150$ & 0 & 0 & 0 & 0 & 0 & \\
\hline Total & $36(18.0)$ & $107(53.5)$ & $54(27.0)$ & $3(1.5)$ & $200(100)$ & \\
\hline
\end{tabular}

${ }^{a}$ Frequencies and percentages along the diagonal self-explanatory--> represent patients whose dose group did not change from first infusion to 10 years following initiation of therapy. Those above the diagonal indicate patients whose dose increased, and those below the diagonal patients whose dose decreased

${ }^{\mathrm{b}}$ Includes 27 patients with partial splenectomy

*Tests of the null hypotheses (i.e., no change from first infusion to 10 years) were calculated using the chi-square testtable->

parameter are not available for every patient, but the collective data set far exceeds any previously described. The ICGG Gaucher Registry has made diligent efforts to verify data as complete and accurate. Patients analyzed in this study are clinically heterogeneous. Once patients have achieved stable disease parameters, their monitoring investigation schedules are generally less intensive, and regular organ imaging does not occur after 8-10 years of treatment, resulting in the relatively small numbers for some parameters in our analysis. Nevertheless, the ICGG Gaucher Registry offers the possibility of evaluating a very large sample of patients with GD1.

Although the relationship between long term effectiveness data and the occurrence of safety and adverse event data are of interest, these were not included in this analysis because data collected through Genzyme's pharmacovigilance system cannot be linked to data in the registry. Safety and adverse event data among Cerezyme users have been previously published and reviewed (Starzyk et al. 2007; Elstein and Zimran 2009).

The patients in this 10-year response study constitute $34.5 \%(N=767)$ of 2,222 registry patients with GD1 with whom alglucerase or imiglucerase was initiated in 1999 or earlier (35.9\% of all patients with intact spleens and $29.7 \%$ of all total splenectomy patients). This study's patients are indistinguishable from the larger registry population in terms of gender, age at diagnosis, and age of first infusion (Supplementary Table 1). Additionally, regardless of splenectomy status, the difference between age at first infusion and at last registry data entry for all 2,222 GD1 patients is 10-12 years, suggesting there is little if any selection bias due to attrition of more severely affected, poorly responding patients. The lack of relevant data at the prescribed 10-year data point was the overwhelming reason for exclusion. Of the 2,222 GD1 patients who began alglucerase/imiglucerase in 1999 or earlier, 149 died [71 of 1,549 (5\%)] intact spleen; 78 of $673(12 \%)$ total splenectomy]. The adequacy of response to treatment in these deceased patients will be evaluated as part of an in-progress mortality analysis.

Splenectomy influences the degree of cytopenia caused by hypersplenism (Hughes et al. 2007) is a recognized risk factor for avascular osteonecrosis (Deegan et al. 2011; Mistry et al. 2009) and is associated with arterial and venous thrombosis and pulmonary arterial hypertension (Crary and Buchanan 2009). Since the advent of imiglucerase therapy, the need for splenectomy to control signs and symptoms of GD1, including severe cytopenias and abdominal discomfort, has been substantially reduced (Cox et al. 2008). To account for the effects of splenectomy on patients with GD1, we analyzed the cohort in two groups. Similar numbers of patients in both groups (nonsplenectomized $47 \%$; splenectomized $48 \%$ ) were diagnosed with GD1 before 10 years of age. The mean age at diagnosis for these two groups was 18 and 15 years, respectively, which is similar to some previous registry reports (Charrow et al. 2000; Weinreb et al. 2002) but younger than a single-center case series and treatment study (Grabowski et al. 1995; Zimran et al. 1992). The differences between reports 
may be due to local genotypic and diagnostic differences, or alternatively, possible selection and reporting bias of registry data (Rothman 2002).

After 10 years of imiglucerase, approximately $90 \%$ of all patients who were anemic at first infusion had normalized hemoglobin levels. We were unable to determine from the registry data whether anemia at the 10 -year point was due to refractory GD or some alternative etiology. At this same time point, $>90 \%(6 / 7)$ of patients who had severe thrombocytopenia at first infusion had improved platelet counts. Despite consistent improvements in thrombocytopenia in patients with intact spleens, proportionately fewer patients had normal platelet counts after 10 years of treatment than patients who had normal hemoglobin concentrations. This observation is consistent with earlier studies of imiglucerase (Weinreb et al. 2002), velaglucerase (Zimran et al. 2010), and taliglucerase (Zimran et al. 2011) and is attributable to multiple possible causes. These causes include differences in platelet and red blood cell (RBC) kinetics; different pooling mechanisms in the spleen; different control mechanisms in the marrow and marrow microenvironment for erythropoiesis and thrombopoiesis, chronic platelet activation, and consumption due to bone disease; differences in the effect of changes in plasma volume on RBC versus platelet concentrations in peripheral blood; presence of antiplatelet antibodies in some patients; and different sensitivity of RBC and platelets to reduction in spleen size (Hollak et al. 2012). All splenectomized patients with thrombocytopenia at first infusion had normal counts at 10 years. The enhanced response in splenectomy patients also reflects previous experience and likely reflects the major contribution of the spleen to the thrombocytopenia due to GD and the relative ease of reversibility of depressed thrombopoiesis in GD-infiltrated marrow. Of patients with severe splenomegaly at first infusion, $97 \%$ had decreased spleen volumes after 10 years. Similar results were reported for liver volumes: all patients who had severe hepatomegaly at first infusion had decreased liver volumes after 10 years.

Genotypes of most of GD1 patients in this study were N370S homozygous or heterozygous, with one of the following mutations: L444P, rare allele, $84 \mathrm{GG}$, or IVS2 +1 . Whereas the L444P/L444P genotype was reported in a few patients (8/493 nonsplenectomized; 4/189 splenectomized), all of those patients were reported by their physicians as having a GD1 phenotype.

Pretreatment hematological and visceral organ abnormalities reported in this study were generally similar to those previously reported by Charrow et al. (2000). Although blood and visceral organ abnormalities are potentially important contributors to morbidity in patients with GD1, hepatosplenomegaly and hematological cytopenias are generally correctable, save for a relatively small number of patients with pretreatment complications, such as fibrotic spleen or liver fibrosis and portal hypertension. On the other hand, skeletal manifestations associated with untreated GD1 have the potential to cause long-term disability and negatively impact the patient's quality of life permanently (Weinreb et al. 2007). In this study, by the time treatment was begun, bone pain was reported by the majority of nonsplenectomized (98/187) and nearly all splenectomized (48/54) patients. Bone crises, which often signal irreversible osteonecrosis, were reported not only in splenectomized (18/47) patients, but also in those with intact spleens (27/169). The percentage of splenectomized patients with bone pain and bone crises was approximately twice that of nonsplenectomized patients ( $89 \%$ vs $52 \%$ and $38 \%$ vs $16 \%$, respectively). Because of the inevitable occurrence of irreversible damage, delay in initiating treatment in patients with bone complications is, as we found, likely to be reflected in a poorer long-term response and clinical outcome.

Previous reports (Grabowski et al. 1998; Pastores et al. 1993; Weinreb et al. 2002) describe the effects of alglucerase/ imiglucerase treatment after 6 months to 5 years. Pastores et al. (1993) and Weinreb et al. (2002) reported improvements in all hematological and visceral organ parameters within the first 6 months to 2 years of therapy. Specifically, Weinreb et al. (2002) reported that patients with anemia achieved normal hemoglobin levels within 2 years of starting imiglucerase, and hemoglobin levels were sustained or increased during the initial 5 years of therapy. In nonsplenectomized patients, the level of platelet response was associated with degree of thrombocytopenia (Weinreb et al. 2002), presence of focal splenic defects (Stein et al. 2010), and dose of imiglucerase (Grabowski et al. 2009). Generally, platelet counts increased during the first 2 years of treatment and continued to improve through 5 years (Weinreb et al. 2002). Similarly, Weinreb et al. (2002) reported decreased spleen and liver volumes with imiglucerase treatment: spleen volumes decreased for the first 2 years, whereas reductions in liver volume may continue to year 5 (Weinreb et al. 2002). Weinreb et al. (2002) noted that liver volumes generally normalized, but decreases in spleen volume to less than five times normal rarely occurred.

A previous report (Wenstrup et al. 2007) indicated that skeletal manifestations, including bone density, took longer ( $\geq 8$ years) to respond to imiglucerase than other hematological and visceral organ manifestations of GD1. Many patients in our study responded positively to imiglucerase treatment, as noted by reported reductions in bone crises and bone pain. Of the nonsplenectomized patients reporting pain at first infusion, $93 \%(25 / 27)$ and $57 \%(56 / 98)$ reported no bone crises nor bone pain, respectively, after 10 years. Decreased bone pain and bone crisis have been reported after the first year of imiglucerase treatment (Charrow et al. 2007; El-Beshlawy et al. 2006; Weinreb et al. 2002). Of the splenectomized patients reporting pain at first infusion, after 10 years, $38 \%$ (18/48) reported no bone pain and $83 \%(15 /$ 18) no bone crises. Splenectomy has been reported to be a 
risk factor for the skeletal manifestations of GD1 (Deegan et al. 2011; Mistry et al. 2009). An interval of $\geq 2$ years between diagnosis and initiation of imiglucerase therapy was associated with a higher risk of severe skeletal complication of avascular osteonecrosis (Mistry et al. 2009). It is possible that other skeletal complications are also related to the time between diagnosis and the start of therapy.

Reports of bone pain to the registry might not necessarily be attributable to GD and may not necessarily be reversible. In addition, it may either be overreported to the registry as limb pain not emanating from bony complications, or misreported as skeletal involvement not related to GD. Similarly, bone crises may be exaggerated if the definition is not strictly adhered to by registry physicians. Such variations may have occurred at first infusion and 10 years after imiglucerase treatment. Because our analysis focused on only two points over a 10 -year observation period (first infusion and 10 years), bone pain results may not accurately portray the patient's chronic state. Despite this possible reporting bias, there was a decrease in bone pain and bone crisis symptoms after 10 years of therapy. Nevertheless, some patients with chronic bone pain may continue to be symptomatic even after years of therapy. Some of these patients may potentially benefit from modern, expert orthopedic interventions.

The clinical responses reported in this study were observed over a broad range of imiglucerase doses at initiation of therapy and at the 10-year period. Response to imiglucerase is statistically related to treatment dose (Grabowski et al. 2009; Wenstrup et al. 2007), although clinically, patients responded over a wide range of doses. In general, after 10 years of therapy, biweekly dose was reduced compared with initial dose. Patients may change dose over time for a number of reasons, including adequate response, compliance, and enzyme availability.

In summary, improvements in hematological, visceral, and bone manifestations previously reported after short-term imiglucerase therapy were maintained after 10 years of treatment in nonsplenectomized and splenectomized patients with GD1.

Acknowledgements We thank patients with type 1 (nonneuronopathic) Gaucher disease and their physicians and health care personnel who submit data to the ICGG Gaucher Registry; the ICGG Gaucher Registry support team at Genzyme; Andrea Gwosdow, Ph.D., for independent data interpretation and drafting the manuscript; John Taylor and Alfred Mbah $\mathrm{Ph} . \mathrm{D}$., for help with analysis; Alison Schroeer for preparing the figures; and Robert Brown and Brian Delguidice for help with tables.

Funding sources Logistical support for this manuscript was provided by Genzyme, a Sanofi company. The database for the International Collaborative Gaucher Group (ICGG) Gaucher Registry is supported by Genzyme.

Competing interests Neal Weinreb receives honoraria and expense reimbursement for serving on a Board of Advisors of the ICGG Gaucher Registry; and travel reimbursements and/or honoraria and/or research support from Genzyme, Shire Pharmaceuticals, Amicus Therapeutics, Protalix Corporation, and Actelion. He has been a study safety board advisor for Synageva Corporation. Jack Goldblatt has received reimbursements and/or honoraria from Genzyme, Shire Pharmaceuticals, Pfizer, Protalix and Actelion. Joel Charrow receives honoraria and expense reimbursement for serving on a Board of Advisors of the ICGG Gaucher Registry and the Fabry Registry and has received consulting fees from Shire Pharmaceuticals and Protalix. Carla Hollak has received honoraria and expense reimbursement for serving on a Board of Advisors of the European ICGG Gaucher Registry, and honoraria and expense reimbursement for lectures from Genzyme, Actelion and Shire Pharmaceuticals. Dr. Hollak donates all honoraria to Gaucher Stichting, a national foundation that supports research in the field of lysosomal storage disorders. Stephan vom Dahl has received honoraria and expense reimbursement for serving on a Board of Advisors of the European ICGG Gaucher Registry, fees for speaking from Genzyme, Shire, Protalix and Actelion and consulting for Actelion. Marcelo Kerstenetzky has received fees for consulting from Genzyme and Biomarin. Jacobo Villalobos has received fees for speaking from Genzyme. J. Alexander Cole is an employee of Genzyme.

Open Access This article is distributed under the terms of the Creative Commons Attribution License which permits any use, distribution, and reproduction in any medium, provided the original author(s) and the source are credited.

\section{References}

Andersson HC, Charrow J, Kaplan P, Mistry P, Pastores GM, PrakashCheng A et al (2005) Individualization of long-term enzyme replacement therapy for Gaucher disease. Genet Med 7:105-110

Barton NW, Brady RO, Dambrosia JM, Di Bisceglie AM, Doppelt SH, Hill SC et al (1991) Replacement therapy for inherited enzyme deficiency - macrophage-targeted glucocerebrosidase for Gaucher's disease. N Engl J Med 324:1464-1470

Biegstraaten M, van Schaik IN, Aerts JM, Hollak CE (2008) 'Nonneuronopathic' Gaucher disease reconsidered. Prevalence of neurological manifestations in a Dutch cohort of type I Gaucher disease patients and a systematic review of the literature. J Inherit Metab Dis 31:337-349

Charrow J, Andersson HC, Kaplan P, Kolodny EH, Mistry P, Pastores $\mathrm{G}$ et al (2000) The Gaucher registry: demographics and disease characteristics of 1698 patients with Gaucher disease. Arch Intern Med 160:2835-2843

Charrow J, Dulisse B, Grabowski GA, Weinreb NJ (2007) The effect of enzyme replacement therapy on bone crisis and bone pain in patients with type 1 Gaucher disease. Clin Genet 71:205-211

Cox TM, Aerts JM, Belmatoug N, Cappellini MD, vom Dahl S, Goldblatt $\mathrm{J}$ et al (2008) Management of non-neuronopathic Gaucher disease with special reference to pregnancy, splenectomy, bisphosphonate therapy, use of biomarkers and bone disease monitoring. J Inherit Metab Dis 31:319-336

Crary SE, Buchanan GR (2009) Vascular complications after splenectomy for hematologic disorders. Blood 114:2861-2868

Deegan PB, Pavlova E, Tindall J, Stein PE, Bearcroft P, Mehta A et al (2011) Osseous manifestations of adult Gaucher disease in the era of enzyme replacement therapy. Med (Baltimore) 90:52-60

El-Beshlawy A, Ragab L, Youssry I, Yakout K, El-Kiki H, Eid K et al (2006) Enzyme replacement therapy and bony changes in Egyptian paediatric Gaucher disease patients. J Inherit Metab Dis 29:92-98

Elstein D, Zimran A (2009) Review of the safety and efficacy of imiglucerase treatment of Gaucher disease. Biologics 3:407-417 
Elstein D, Hadas-Halpern I, Azuri Y, Abrahamov A, Bar-Ziv Y, Zimran A (1997) Accuracy of ultrasonography in assessing spleen and liver size in patients with Gaucher disease: comparison to computed tomographic measurements. J Ultrasound Med 16:209-211

Grabowski GA, Barton NW, Pastores G, Dambrosia JM, Banerjee TK, McKee MA et al (1995) Enzyme therapy in type 1 Gaucher disease: comparative efficacy of mannose-terminated glucocerebrosidase from natural and recombinant sources. Ann Intern Med 122:33-39

Grabowski GA, Leslie N, Wenstrup R (1998) Enzyme therapy for Gaucher disease: the first 5 years. Blood Rev 12:115-133

Grabowski GA, Kacena K, Cole JA, Hollak CE, Zhang L, Yee J et al (2009) Dose-response relationships for enzyme replacement therapy with imiglucerase/alglucerase in patients with Gaucher disease type 1. Genet Med 11:92-100

Grabowski G, Kolodny E, Weinreb N, Rosenbloom B, Prakash-Cheng A, Kaplan P et al (2010) Gaucher disease: Phenotypic and genetic variation. In C. Scriver, A. Beaudet, D. Valle and W. Slye, eds. The Online Metabolic and Molecular Basis of Inherited Metabolic Disease. New York: McGraw-Hill Publishers. <http:// genetics.accessmedicine.com/server-java/Arknoid/amed/ mmbid/co_chapters/ch146.1/146.1_p01.html> Access Date: 1 Sept 2011

Hollak CE, Aerts JM, Ayme S, Manuel J (2011) Limitations of drug registries to evaluate orphan medicinal products for the treatment of lysosomal storage disorders. Orphanet J Rare Dis 6:16

Hollak CE, Belmatoug N, Alexander Cole J, Vom Dahl S, Deegan PB, Goldblatt J et al (2012) Characteristics of type I Gaucher disease associated with persistent thrombocytopenia after treatment with imiglucerase for 4-5 years. Br J Haematol 158:528-538

Hughes D, Cappellini MD, Berger M, Van Droogenbroeck J, de Fost M, Janic D et al (2007) Recommendations for the management of the haematological and onco-haematological aspects of Gaucher disease. Br J Haematol 138:676-686

Kaplan P, Andersson HC, Kacena KA, Yee JD (2006) The clinical and demographic characteristics of nonneuronopathic Gaucher disease in 887 children at diagnosis. Arch Pediatr Adolesc Med 160:603-608

Ludwig J (1979) Current methods of autopsy practice. W.B. Saunders Company, Philadelphia

Mistry PK, Deegan P, Vellodi A, Cole JA, Yeh M, Weinreb NJ (2009) Timing of initiation of enzyme replacement therapy after diagnosis of type 1 Gaucher disease: effect on incidence of avascular necrosis. Br J Haematol 147:561-570

Pastores GM, Sibille AR, Grabowski GA (1993) Enzyme therapy in Gaucher disease type 1: dosage efficacy and adverse effects in 33 patients treated for 6 to 24 months. Blood $82: 408-416$

Rothman K (2002) Epidemiology: an introduction. Oxford University Press, New York

Sims KB, Pastores GM, Weinreb NJ, Barranger J, Rosenbloom BE, Packman $S$ et al (2008) Improvement of bone disease by imiglucerase (Cerezyme) therapy in patients with skeletal manifestations of type 1 Gaucher disease: results of a 48-month longitudinal cohort study. Clin Genet 73:430-440

Starzyk K, Richards S, Yee J, Smith SE, Kingma W (2007) The longterm international safety experience of imiglucerase therapy for Gaucher disease. Mol Genet Metab 90:157-163

Stein P, Malhotra A, Haims A, Pastores GM, Mistry PK (2010) Focal splenic lesions in type I Gaucher disease are associated with poor platelet and splenic response to macrophage-targeted enzyme replacement therapy. J Inherit Metab Dis 33:769-774

Weinreb NJ, Charrow J, Andersson HC, Kaplan P, Kolodny EH, Mistry P et al (2002) Effectiveness of enzyme replacement therapy in 1028 patients with type 1 Gaucher disease after 2 to 5 years of treatment: a report from the Gaucher Registry. Am J Med 113:112-119

Weinreb N, Barranger J, Packman S, Prakash-Cheng A, Rosenbloom B, Sims K et al (2007) Imiglucerase (Cerezyme) improves quality of life in patients with skeletal manifestations of Gaucher disease. Clin Genet 71:576-588

Weinreb N, Taylor J, Cox T, Yee J, vom Dahl S (2008) A benchmark analysis of the achievement of therapeutic goals for type 1 Gaucher disease patients treated with imiglucerase. Am J Hematol 83:890-895

Wenstrup RJ, Kacena KA, Kaplan P, Pastores GM, Prakash-Cheng A, Zimran A et al (2007) Effect of enzyme replacement therapy with imiglucerase on BMD in type 1 Gaucher disease. J Bone Miner Res 22:119-126

Zimran A, Kay A, Gelbart T, Garver P, Thurston D, Saven A et al (1992) Gaucher disease. Clinical, laboratory, radiologic, and genetic features of 53 patients. Med (Baltimore) 71:337-353

Zimran A, Altarescu G, Philips M, Attias D, Jmoudiak M, Deeb M et al (2010) Phase $1 / 2$ and extension study of velaglucerase alfa replacement therapy in adults with type 1 Gaucher disease: 48month experience. Blood 115:4651-4656

Zimran A, Brill-Almon E, Chertkoff R, Petakov M, Blanco-Favela F, Munoz ET et al (2011) Pivotal trial with plant cell-expressed recombinant glucocerebrosidase, taliglucerase alfa, a novel enzyme replacement therapy for Gaucher disease. Blood 118:5767-5773 